\title{
Coherent states, Path integral, and Semiclassical approximation
}

\author{
Kunio FUNAHASHI, Taro KASHIWA, and Seiji SAKODA \\ Department of Physics, Kyushu University,Fukuoka 812, Japan
}

Kazuyuki FUJII

Department of Mathematics, Yokohama City University, Yokohama 236, Japan

(June 30, 2018)

\begin{abstract}
Using the generalized coherent states we argue that the path integral formulae for $S U(2)$ and $S U(1,1)$ (in the discrete series) are WKB exact, if the starting point is expressed as the trace of $e^{-i T \hat{H}}$ with $\hat{H}$ being given by a linear combination of generators. In our case, WKB approximation is achieved by taking a large "spin" limit: $J, K \rightarrow \infty$. The result is obtained directly by knowing that the each coefficient vanishes under the $J^{-1}\left(K^{-1}\right)$ expansion and is examined by another method to be legitimated. We also point out that the discretized form of path integral is indispensable, in other words, the continuum path integral expression leads us to a wrong result. Therefore a great care must be taken when some geometrical action would be adopted, even if it is so beautiful, as the starting ingredient of path integral.
\end{abstract}

Typeset using REVTEX 


\section{INTRODUCTION}

Physical systems in actual situation are so complicated that even in a simplified form there usually need some approximation techniques, such as perturbation of the coupling constant or the self-consistent manner of Hartree-Fock. In path integral the most promising one seems to be a semiclassical (WKB) approximation. However there are some systems in which the WKB approximation gives the exact result: a harmonic oscillator is the well-known example (and may be the only case if we are in a usual quantum mechanical circumstance, that is, on a flat and non-compact manifold); since the Hamiltonian is quadratic in momenta and coordinates, yielding a Gaussian form in the path integral.

If generalization is made to quantum mechanics on a non-trivial manifold such as $S U(2)$ spin system [1] [2] [3] or $\mathbf{C} P^{N}$ system, a new possibility may occur [4]: this new possibility of exactness of the WKB approximation is discussed in connection with the theorem of Duistermaat-Heckman(D-H) [5] and is extended to Grassmannian manifold [6]. So far the discussion has been concentrated mainly on the geometrical view point [7]. However there are a number of unsatisfactory points and mysteries: the ingredient for obtaining path integral formulae is (apart from [1], [2], and [3]) so called (generalized) coherent states. They have employed naive calculus, $\left\langle g \mid g^{\prime}\right\rangle \sim 1+\langle g \mid \delta g\rangle \sim \exp \langle g \mid \delta g\rangle$, where $|g\rangle$ is some generalized coherent states and the elements $g$ and $g^{\prime}$ are assumed to $g^{\prime}=g+\delta g$ with

$\delta g \ll 1$; which, however, cannot be justified because $g$ and $g^{\prime}$ are the integration variables in the path integral. Thus obtained "quantum" action in the path integral formula has already been in a semiclassical state. The reason why this kind of rough estimation could have been accepted is that the resultant action is beautifully geometric. In this sense, it is still unsatisfactory for us that ad hoc adoption of geometrical actions in path integral even if those would give us a correct result.

Another issue in our mind is that we should be so careful in performing WKB approximation: first point is so called overspecification problem. It is often said that the WKB approximation is not allowed in the case of kernel under the (canonical) coherent state 
representation;

$$
\begin{gathered}
a|z\rangle=z|z\rangle ; \quad|z\rangle \equiv \exp \left(-\frac{1}{2}|z|^{2}+z a^{\dagger}\right)|0\rangle, \quad(a|0\rangle=0) \\
\left\langle z \mid z^{\prime}\right\rangle=\exp \left(-\frac{1}{2}|z|^{2}-\frac{1}{2}\left|z^{\prime}\right|^{2}+z^{*} z^{\prime}\right) \\
\int \frac{d z^{*} d z}{\pi}|z\rangle\langle z|=\mathbf{1}
\end{gathered}
$$

giving

$$
\begin{gathered}
K\left(z_{F}, z_{I} ; T\right) \equiv\left\langle z_{F}\left|e^{-i T H\left(a^{\dagger}, a\right)}\right| z_{I}\right\rangle=\lim _{N \rightarrow \infty}\left\langle z_{F}\left|\left(1-i \epsilon H\left(a^{\dagger}, a\right)\right)^{N}\right| z_{I}\right\rangle \quad\left(\epsilon \equiv \frac{T}{N}\right) \\
=\lim _{N \rightarrow \infty} \prod_{j=1}^{N-1} \int \frac{d z_{j}^{*} d z_{j}}{\pi} \exp \left[-\sum_{j=1}^{N}\left[\frac{1}{2}\left\{z_{j}^{*}\left(z_{j}-z_{j-1}\right)-\left(z_{j}^{*}-z_{j-1}^{*}\right) z_{j-1}\right\}+i \epsilon H\left(z_{j}^{*}, z_{j-1}\right)\right]\right] \\
\left(\text { with } z_{0}=z_{I}, z_{N}=z_{F}\right)
\end{gathered}
$$

where we have successively inserted the resolution of unity (1.2) into the product in third expression of the first line. The WKB-approximation is performed by putting $z_{j}=z_{j}^{c}+z_{j}^{\prime}$, where $z_{j}^{c}$ 's satisfy the classical continuum equations of motion, $i \partial z^{c}(t) / \partial t=$ $\partial H\left(z^{*}, z\right) / \partial z^{c}(t)^{*}$; since $z_{j}^{c}$ 's are not the integration variables so that the continuum limit can be taken safely. However there are two boundary conditions ; $z^{c}(T)=z_{F}, z^{c}(0)=z_{I}$ despite the fact that the equations of motion are first order differential equations [8] F. For the trace formula,

\footnotetext{
${ }^{1}$ On the other hand, when applied to the discrete time formulation given by the second line of (1.3), WKB approximation is nothing but a saddle point approximation for $N-1$ integration variables. For this case we have a set of $2(N-1)$ equations

$$
i\left(z_{j}-z_{j-1}\right)=\frac{\partial}{\partial z_{j}^{*}} H\left(z_{j}^{*}, z_{j-1}\right),-i\left(z_{j+1}^{*}-z_{j}^{*}\right)=\frac{\partial}{\partial z_{j}} H\left(z_{j+1}^{*}, z_{j}\right) \quad(1 \leq j \leq N-1) .
$$

They should be solved with conditions $z_{0}=z_{I}$ for the first equation and $z_{N}^{*}=z_{F}^{*}$ for the second one respectively. Thus we see there is no such problem if we work with the discrete time formulation of path integral.
} 


$$
\begin{aligned}
\operatorname{Tr} e^{-i T H\left(a^{\dagger}, a\right)} & =\int \frac{d z^{*} d z}{\pi}\left\langle z\left|e^{-i T H\left(a^{\dagger}, a\right)}\right| z\right\rangle \\
& =\lim _{N \rightarrow \infty} \prod_{j=1}^{N} \int_{\mathrm{PBC}} \frac{d z_{j}^{*} d z_{j}}{\pi} \exp \left\{-z_{j}^{*}\left(z_{j}-z_{j-1}\right)-i \epsilon H\left(z_{j}^{*}, z_{j-1}\right)\right\},
\end{aligned}
$$

the boundary condition becomes a periodic one; $z_{N}=z_{0}(\mathrm{PBC})$, which has of course no problem. Case is unchanged for the generalized coherent state representation; however, seems a little bit drastic in $S U(2)$ of Nielsen-Rohrlich formula [1], [2], [3]: the boundary condition is $\phi(T)=\phi(0)+2 n \pi(n \in \mathbf{Z})$, but the equation of motion reads $\dot{\phi}(t)=h(h$ : constant). They are never compatible. (See the discussion.)

The second point that we would like to mention is subtlety of the use of continuum path integral under the coherent representation: as an example, take the harmonic oscillator, $H=\omega a^{\dagger} a$, in (1.4) and go to the naive continuum limit, giving

$$
\begin{aligned}
\operatorname{Tr} e^{-i \omega a^{\dagger} a T} & =\int_{\mathrm{PBC}} \mathcal{D} z^{*} \mathcal{D} z \exp \left\{-\int_{0}^{T} d t\left(z^{*} \dot{z}+i \omega z^{*} z\right)\right\} \\
& \equiv \operatorname{det}\left(\frac{d}{d t}+i \omega\right)^{-1}=\frac{1}{2 i \sin (\omega T / 2)}
\end{aligned}
$$

where the determinant has been defined as, $\operatorname{det}(d / d t+i \omega) \equiv \prod_{n=-\infty}^{\infty}(i 2 n \pi / T+i \omega)$, with $i 2 n \pi / T$ coming from a periodic eigenfunction; $f_{n}(t)=e^{i 2 n \pi / T} / \sqrt{T}$, and use has been made of the $\zeta$-function regularization. The correct answer is

$$
\operatorname{Tr} e^{-i \omega a^{\dagger} a T}=\frac{1}{1-e^{-i \omega T}}=\frac{e^{i \omega T / 2}}{2 i \sin (\omega T / 2)},
$$

of course. This discrepancy when being in the continuum expression is always left in other cases such as $S U(2)$ and might be in the Chern-Simons field theory as a need for the Coxeter number [9](see the discussion).

Motivated by these, we shall in this paper study the exactness of the WKB approximation under the path integral formula in the case of $S U(2)$ and $S U(1,1)$ with the aid of generalized coherent states. To avoid the above questionable issues, we shall concentrate only on formulae like (1.4). In section 2, we shall introduce the path integral expression for the character formulae of $S U(2)$ and $S U(1,1)$ and study their structure. The following 
section 3 deals with the exactness of the WKB approximation. The final section will be devoted to discussions and in the appendix, geometrical properties of generalized coherent states and the relationship to the canonical coherent states will be presented, which will be useful for an analysis of $\mathbf{C} P^{N}$ as well as Grassmannian manifold.

\section{COHERENT STATES AND PATH INTEGRAL FORMULAE \\ FOR $S U(2)$ AND $S U(1,1)$}

\section{A. $S U(2)$;}

$s u(2)$ algebra reads

$$
\left[J_{+}, J_{-}\right]=2 J_{3}, \quad\left[J_{3}, J_{ \pm}\right]= \pm J_{ \pm}
$$

where $J_{ \pm} \equiv J_{1} \pm i J_{2}$. Take a representation as usual,

$$
J_{3}|J, M\rangle=M|J, M\rangle, J_{ \pm}|J, M\rangle=\sqrt{(J \mp M)(J \pm M+1)}|J, M \pm 1\rangle, \quad(|M| \leq J) .
$$

Let us define

$$
\left.\mid \xi) \equiv e^{\xi J_{+}}|J,-J\rangle, \quad|\xi\rangle \equiv \frac{1}{(\xi \mid \xi)^{1 / 2}} \mid \xi\right) \quad, \quad \xi \in \mathbf{C}
$$

where we have introduced $|J,-J\rangle$ as the fiducial vector [10]. Explicitly

$$
\mid \xi)=\sum_{m=0}^{2 J} \xi^{m}\left(\begin{array}{c}
2 J \\
m
\end{array}\right)^{1 / 2}|J,-J+m\rangle
$$

and whose norm is found to be $(\xi \mid \xi)=\left(1+|\xi|^{2}\right)^{2 J}$, giving the normalized state,

$$
|\xi\rangle=\frac{1}{\left(1+|\xi|^{2}\right)^{J}} \sum_{m=0}^{2 J} \xi^{m}\left(\begin{array}{c}
2 J \\
m
\end{array}\right)^{1 / 2}|J,-J+m\rangle
$$

They satisfy

$$
\left\langle\xi \mid \xi^{\prime}\right\rangle=\frac{\left(1+\xi^{*} \xi^{\prime}\right)^{2 J}}{\left(1+|\xi|^{2}\right)^{J}\left(1+\left|\xi^{\prime}\right|^{2}\right)^{J}}
$$




$$
\frac{2 J+1}{\pi} \int \frac{d \xi^{*} d \xi}{\left(1+|\xi|^{2}\right)^{2}}|\xi\rangle\left\langle\xi\left|\equiv \int d \mu\left(\xi^{*}, \xi\right)\right| \xi\right\rangle\langle\xi|=\mathbf{1}_{J}
$$

where

$$
d \xi^{*} d \xi \equiv d \operatorname{Re}(\xi) d \operatorname{Im}(\xi)
$$

and

$$
\mathbf{1}_{J} \equiv \sum_{M=-J}^{J}|J, M\rangle\langle J, M|
$$

is the identity operator in $2 J+1$-dimensional irreducible representation. Matrix elements of generators are found to be

$$
\begin{aligned}
& \left\langle\xi\left|J_{3}\right| \xi^{\prime}\right\rangle=-J \frac{1-\xi^{*} \xi^{\prime}}{1+\xi^{*} \xi^{\prime}}\left\langle\xi \mid \xi^{\prime}\right\rangle, \\
& \left\langle\xi\left|J_{+}\right| \xi^{\prime}\right\rangle=J \frac{2 \xi^{*}}{1+\xi^{*} \xi^{\prime}}\left\langle\xi \mid \xi^{\prime}\right\rangle .
\end{aligned}
$$

Armed with these machineries, we can now discuss the path integral formula for a Hamiltonian

$$
H=h_{1} J_{1}+h_{2} J_{2}+h_{3} J_{3} \in s u(2)
$$

The starting point is the trace formula

$$
Z(T) \equiv \operatorname{Tr} e^{-i H T}=\operatorname{Tr} \lim _{N \rightarrow \infty}(\mathbf{1}-i \epsilon H)^{N}=\lim _{N \rightarrow \infty} Z_{N}
$$

where

$$
Z_{N} \equiv \prod_{j=1}^{N} \int_{\mathrm{PBC}} d \mu\left(\xi_{j}^{*}, \xi_{j}\right) \exp \left[i\left\{-i \ln \left\langle\xi_{j} \mid \xi_{j-1}\right\rangle-\epsilon \mathcal{H}\left(\xi_{j}^{*}, \xi_{j-1}\right)\right\}\right]
$$

with $d \mu\left(\xi_{j}^{*}, \xi_{j}\right)$ being given by $(2.7)$ and

$$
\mathcal{H}\left(\xi_{j}^{*}, \xi_{j-1}\right) \equiv \frac{\left\langle\xi_{j}|H| \xi_{j-1}\right\rangle}{\left\langle\xi_{j} \mid \xi_{j-1}\right\rangle}
$$

Here as in the introduction we have repeatedly inserted the resolution of unity (2.7) into the second relation in (2.12). Within the trace (2.12), we always get a diagonalized Hamiltonian by use of $S U(2)$ rotation 


$$
U H U^{\dagger}=h J_{3}, \quad U \in S U(2),
$$

thus (2.12) is equivalent to the character formula;

$$
Z(T)=\operatorname{Tr} \exp \left(-i h J_{3} T\right)=\frac{\sin ((J+1 / 2) h T)}{\sin (h T / 2)} .
$$

Therefore, with the aid of (2.10), (2.13) is found to be

$$
Z_{N}=\prod_{j=1}^{N} \int_{\mathrm{PBC}} d \mu\left(\xi_{j}^{*}, \xi_{j}\right) \exp \left[i J\left\{2 i \ln \left(\frac{1+\xi_{j}^{*} \xi_{j}}{1+\xi_{j}^{*} \xi_{j-1}}\right)+\epsilon h \frac{1-\xi_{j}^{*} \xi_{j-1}}{1+\xi_{j}^{*} \xi_{j-1}}\right\}\right] .
$$

In the following we shall consider the case that $J$ becomes large; where the saddle point of the exponent in (2.17) is important, which is given by a set of equations,

$$
\frac{\xi_{j}-\xi_{j-1}}{1+\xi_{j}^{*} \xi_{j}}=\frac{-i \epsilon h \xi_{j-1}}{1+\xi_{j}^{*} \xi_{j-1}}, \quad \frac{\xi_{j}^{*}-\xi_{j+1}^{*}}{1+\xi_{j}^{*} \xi_{j}}=\frac{-i \epsilon h \xi_{j+1}}{1+\xi_{j+1}^{*} \xi_{j}} \quad(1 \leq j \leq N) .
$$

There are two solutions, satisfying PBC;

$$
\xi_{j}^{c}=0 \equiv \xi_{c}^{(+)}, \quad \xi_{j}^{c}=\infty \equiv \xi_{c}^{(-)}
$$

whose (finite!) contribution to the exponent (2.17) is read as $e^{i h J T}\left(e^{-i h J T}\right)$ for $\xi_{c}^{(+)}\left(\xi_{c}^{(-)}\right)$.

By knowing that the measure $d \mu\left(\xi^{*}, \xi\right)$ in (2.7) is invariant under $\xi \mapsto 1 / \xi$, and that $\prod_{j=1}^{N}\left(\xi_{j} / \xi_{j-1}\right)=1$ under PBC, the change of variable, $\xi_{j} \mapsto 1 / \xi_{j}$, brings (2.17) to

$$
Z_{N}=\prod_{j=1}^{N} \int_{\mathrm{PBC}} d \mu\left(\xi_{j}^{*}, \xi_{j}\right) \exp \left[i J\left\{2 i \ln \left(\frac{1+\xi_{j}^{*} \xi_{j}}{1+\xi_{j}^{*} \xi_{j-1}}\right)-\epsilon h \frac{1-\xi_{j}^{*} \xi_{j-1}}{1+\xi_{j}^{*} \xi_{j-1}}\right\}\right] .
$$

Apparently the two expressions are related by $h \mapsto-h$.

\section{B. $S U(1,1)$;}

$s u(1,1)$ algebra is given by

$$
\left[K_{1}, K_{2}\right]=-i K_{3}, \quad\left[K_{2}, K_{3}\right]=i K_{1}, \quad\left[K_{3}, K_{1}\right]=i K_{2},
$$

with $\mathbf{K}^{2}=K_{1}^{2}+K_{2}^{2}-K_{3}^{2}$, or

$$
\left[K_{+}, K_{-}\right]=2 K_{3}, \quad\left[K_{3}, K_{ \pm}\right]= \pm K_{ \pm} ; \quad K_{ \pm} \equiv \pm\left(K_{1} \pm i K_{2}\right) ;\left(K_{+}\right)^{\dagger}=-K_{-}
$$


with $\mathbf{K}^{2}=-K_{+} K_{-}-K_{3}^{2}+K_{3}=-K_{-} K_{+}-K_{3}^{2}-K_{3}$.

We confine ourselves in a discrete series [1] to write

$$
\begin{aligned}
\mathbf{K}^{2}|K, M\rangle & =K(1-K)|K, M\rangle, \quad K_{3}|K, M\rangle=M|K, M\rangle \\
K_{ \pm}|K, M\rangle & = \pm \sqrt{(M \pm K)(M \mp K \pm 1)}|K, M \pm 1\rangle \\
M & =K, K+1, K+2, \ldots \quad, \quad K=1 / 2,1,3 / 2,2, \ldots .
\end{aligned}
$$

Adopting $|K, K\rangle$ as the fiducial vector, $K_{-}|K, K\rangle=0$, as before, we obtain

$$
\begin{aligned}
\mid \xi) & \equiv e^{\xi K_{+}}|K, K\rangle=\sum_{m=0}^{\infty} \xi^{m}\left(\begin{array}{c}
2 K+m-1 \\
m
\end{array}\right)^{1 / 2}|K, K+m\rangle, \\
\xi & \in D_{(1,1)}=\{\xi \in \mathbf{C},|\xi|<1\} .
\end{aligned}
$$

Their inner product is found to be

$$
\begin{aligned}
\left(\xi \mid \xi^{\prime}\right) & =\sum_{m=0}^{\infty}\left(\xi^{*} \xi^{\prime}\right)^{2 m}\left(\begin{array}{c}
2 K+m-1 \\
m
\end{array}\right) \\
& =\sum_{m=0}^{\infty} \frac{\left(\xi^{*} \xi^{\prime}\right)^{2 m}}{m !} \frac{1}{\Gamma(2 K)} \int_{0}^{\infty} d x x^{2 K+m-1} e^{-x} \\
& =\left(\frac{1}{1-\xi^{*} \xi^{\prime}}\right)^{2 K}
\end{aligned}
$$

where we have introduced the integral representation of $\Gamma$-function,

$$
\int_{0}^{\infty} d x x^{n-1} e^{-x}=\Gamma(n),
$$

in the second line, then performed the summation with respect to $m$ and used (2.26) again in the final expression. Thus the normalized state is given as

$$
\begin{aligned}
|\xi\rangle & \left.\equiv \frac{1}{(\xi \mid \xi)^{1 / 2}} \mid \xi\right) \\
& =\left(1-|\xi|^{2}\right)^{K} \sum_{m=0}^{\infty} \xi^{m}\left(\begin{array}{c}
2 K+m-1 \\
m
\end{array}\right)^{1 / 2}|K, K+m\rangle,
\end{aligned}
$$

whose inner product and the resolution of unity in this case read as

$$
\left\langle\xi \mid \xi^{\prime}\right\rangle=\frac{\left(1-|\xi|^{2}\right)^{K}\left(1-\left|\xi^{\prime}\right|^{2}\right)^{K}}{\left(1-\xi^{*} \xi^{\prime}\right)^{2 K}},
$$




$$
\frac{2 K-1}{\pi} \int_{D_{(1,1)}} \frac{d \xi^{*} d \xi}{\left(1-|\xi|^{2}\right)^{2}}|\xi\rangle\left\langle\xi\left|\equiv \int_{D_{(1,1)}} d \mu\left(\xi^{*}, \xi\right)\right| \xi\right\rangle\langle\xi|=\mathbf{1}_{K}
$$

where $\mathbf{1}_{K} \equiv \sum_{m=0}^{\infty}|K, K+m\rangle\langle K, K+m|$. (It should be noted that regularization for the measure of $K=1 / 2$ is needed. See Appendix A.)

Matrix elements of generators are obtained as

$$
\begin{aligned}
& \left\langle\xi\left|K_{3}\right| \xi^{\prime}\right\rangle=K \frac{1+\xi^{*} \xi^{\prime}}{1-\xi^{*} \xi^{\prime}}\left\langle\xi \mid \xi^{\prime}\right\rangle \\
& \left\langle\xi\left|K_{+}\right| \xi^{\prime}\right\rangle=K \frac{2 \xi^{*}}{1-\xi^{*} \xi^{\prime}}\left\langle\xi \mid \xi^{\prime}\right\rangle
\end{aligned}
$$

Now we build up the path integral formula: again start with the trace formula

$$
\begin{aligned}
Z(T) & =\operatorname{Tr} e^{-i H T} \\
& =\sum_{m=0}^{\infty}\left\langle K, K+m\left|e^{-i H T}\right| K, K+m\right\rangle \quad \text { (in ortho-normal basis) } \\
& =\int d \mu(\xi)\left\langle\xi\left|e^{-i H T}\right| \xi\right\rangle=\lim _{N \rightarrow \infty} Z_{N} \quad \text { (in coherent basis) }
\end{aligned}
$$

with the Hamiltonian of the form

$$
H=h_{1} K_{1}+h_{2} K_{2}-h_{3} K_{3}, \quad h_{1}^{2}+h_{2}^{2}-h_{3}^{2}<0
$$

which can be diagonalized as

$$
V^{-1} H V=h K_{3}, \quad V \in S U(1,1)
$$

Therefore $Z_{N}$ in 2.30 is given by

$$
Z_{N}=\prod_{j=1}^{N} \int_{\mathrm{PBC}} d \mu\left(\xi_{j}^{*}, \xi_{j}\right) \exp \left[i K\left\{2 i \ln \left(\frac{1-\xi_{j}^{*} \xi_{j-1}}{1-\xi_{j}^{*} \xi_{j}}\right)-\epsilon h \frac{1+\xi_{j}^{*} \xi_{j-1}}{1-\xi_{j}^{*} \xi_{j-1}}\right\}\right] .
$$

It should be noted that $\xi$ 's are in $D_{(1,1)}$ and the spectrum in the trace formula (2.30) is unbounded in this case while bounded in the $S U(2)$ case.

Now under $K$ being large, consider the saddle point conditions,

$$
\frac{\xi_{j}-\xi_{j-1}}{1-\xi_{j}^{*} \xi_{j}}=\frac{-i \epsilon h \xi_{j-1}}{1-\xi_{j}^{*} \xi_{j-1}}, \quad \frac{\xi_{j}^{*}-\xi_{j+1}^{*}}{1-\xi_{j}^{*} \xi_{j}}=\frac{-i \epsilon h \xi_{j+1}}{1-\xi_{j+1}^{*} \xi_{j}} \quad(1 \leq j \leq N)
$$

which has only one solution, 


$$
\xi_{j}^{c}=0 \equiv \xi_{c}^{(0)}
$$

whose value of the exponent in (2.33) is $e^{-i h K T}$, because the other solution, $\xi=\infty$, for (2.34) is outside of $D_{(1,1)}$.

\section{EXACTNESS OF THE WKB APPROXIMATION}

The WKB approximation is valid as the saddle point method when $J(K)$ becomes large in $S U(2)(S U(1,1))$ case. To make the expansion transparent, let us put

$$
\xi_{j}=\sqrt{\kappa} z_{j}
$$

in $(2.17),(2.20)$, and $(2.33)$ respectively. Here

$$
\kappa \equiv\left\{\begin{array}{l}
\frac{1}{2 J+1}: S U(2) \\
\frac{1}{2 K-1}: S U(1,1) .
\end{array}\right.
$$

It would be needless to say that the expression (2.20) is suitable when expanding around $\xi=\infty$. Plugging (3.1) into (2.17), (2.20), and (2.33) and expanding the logarithm, we have

$$
\begin{aligned}
Z_{N}^{(\alpha)}\left(\kappa^{(\alpha)}\right) \equiv & e^{i h^{(\alpha)} J^{(\alpha)} T} \prod_{j=1}^{N} \int \frac{d z_{j}^{*} d z_{j}}{\pi} \exp \left[-\left(z_{j}^{*} z_{j}-e^{-i \epsilon h^{(\alpha)}} z_{j}^{*} z_{j-1}\right)\right. \\
& +\sum_{n=1}^{\infty} \frac{(-)^{n}}{n}\left(\kappa^{(\alpha)}\right)^{n}\left[\left\{\left(z_{j}^{*} z_{j}\right)^{n}+\left(e^{-i \epsilon h^{(\alpha)}} z_{j}^{*} z_{j-1}\right)^{n}\right\}\right. \\
& \left.\left.-\frac{n}{n+1}\left\{\left(z_{j}^{*} z_{j}\right)^{n+1}-\left(e^{-i \epsilon h^{(\alpha)}} z_{j}^{*} z_{j-1}\right)^{n+1}\right\}\right]\right],
\end{aligned}
$$

where we have discarded $O\left(\epsilon^{2}\right)$ terms to arrange the expression and $\alpha$ takes the value + , -, and 0 corresponding to (2.17), (2.20), and (2.33) respectively. (Recall the label of saddle point solutions; 2.19) and (2.35).) Thus

$$
\left(h^{( \pm)}, h^{(0)}\right) \equiv( \pm h, h) ; \quad\left(J^{( \pm)}, J^{(0)}\right) \equiv(J, K) ; \quad\left(\kappa^{( \pm)}, \kappa^{(0)}\right) \equiv(\kappa,-\kappa)
$$

In terms of $Z_{N}^{(\alpha)}\left(\kappa^{(\alpha)}\right), Z_{N}$ is given, when $J$ or $K \rightarrow \infty$,

$$
\begin{array}{lll}
Z_{N}=Z_{N}^{(+)}\left(\kappa^{(+)}\right)+Z_{N}^{(-)}\left(\kappa^{(-)}\right) & : & S U(2), \\
Z_{N}=Z_{N}^{(0)}\left(\kappa^{(0)}\right) & : & S U(1,1) .
\end{array}
$$


Therefore if the path integral in $S U(2)$ or $S U(1,1)$ would be WKB-exact, the target is to prove the relation;

$$
Z_{N}(\kappa)=Z_{N}(0)=e^{i h J T} \prod_{j=1}^{N} \int \frac{d z_{j}^{*} d z_{j}}{\pi} \exp \left\{-\left(z_{j}^{*} z_{j}-e^{-i \epsilon h} z_{j}^{*} z_{j-1}\right)\right\}
$$

for any $\alpha$. (Thus we have omitted the superscript $\alpha$.) To this end, introduce parameters, $a_{j}$ 's and $b_{j}$ 's, then write

$$
\begin{aligned}
Z_{N}(\kappa)= & \exp \left[\sum _ { n = 1 } ^ { \infty } \frac { ( - ) ^ { n } } { n } \kappa ^ { n } \sum _ { j = 1 } ^ { N } \left[\left\{\left(-\frac{\partial}{\partial a_{j}}\right)^{n}+\left(\frac{\partial}{\partial b_{j}}\right)^{n}\right\}\right.\right. \\
& \left.\left.-\frac{n}{n+1}\left\{\left(-\frac{\partial}{\partial a_{j}}\right)^{n+1}-\left(\frac{\partial}{\partial b_{j}}\right)^{n+1}\right\}\right]\right] \\
& \times\left.\prod_{j=1}^{N} \int \frac{d z_{j}^{*} d z_{j}}{\pi} \exp \left\{-\left(a_{j} z_{j}^{*} z_{j}-b_{j} e^{-i \epsilon h} z_{j}^{*} z_{j-1}\right)\right\}\right|_{\{a\}=\{b\}=1} \\
= & \left.\prod_{i=1}^{N} F\left(\kappa ;-\partial_{a_{i}}, \partial_{b_{i}}\right) \int_{0}^{\infty} d \tau G((\mathbf{a}-\gamma \mathbf{b}) \tau)\right|_{\{a\}=\{b\}=1}, \quad \gamma \equiv e^{-i h T},
\end{aligned}
$$

where

$$
F(\kappa ; x, y) \equiv \exp \left[\sum_{n=1}^{\infty} \frac{(-1)^{n}}{n} \kappa^{n}\left\{\left(x^{n}+y^{n}\right)-\frac{n}{n+1}\left(x^{n+1}-y^{n+1}\right)\right\}\right]
$$

and, for later convinience, we have introduced

$$
G((\mathbf{a}-\gamma \mathbf{b}) \tau) \equiv \exp \{-(\mathbf{a}-\gamma \mathbf{b}) \tau\}
$$

since

$$
\begin{aligned}
& \prod_{j=1}^{N} \int \frac{d z_{j}^{*} d z_{j}}{\pi} \exp \left\{-\left(a_{j} z_{j}^{*} z_{j}-e^{-i \epsilon h} b_{j} z_{j}^{*} z_{j-1}\right)\right\}=\frac{1}{\prod_{j=1}^{N} a_{j}-\gamma \prod_{j=1}^{N} b_{j}} \equiv \frac{1}{\mathbf{a}-\gamma \mathbf{b}} \\
& =\int_{0}^{\infty} d \tau G((\mathbf{a}-\gamma \mathbf{b}) \tau) .
\end{aligned}
$$

(Thus the condition, $\operatorname{Re}(\mathbf{a}-\gamma \mathbf{b})>0$, should be understood.) Here $a$ 's and $b$ 's have been put unity after all, designating $\{a\}=\{b\}=1\left(\equiv\left\{a_{1}=b_{1}=\cdots=a_{N}=b_{N}=1\right\}\right)$.

The important ingredient for the proof 


\section{Formula;}

$$
\left.F\left(\kappa ;-\partial_{a_{i}}, \partial_{b_{i}}\right) \int_{0}^{\infty} d \tau G((\mathbf{a}-\gamma \mathbf{b}) \tau)\right|_{a_{i}=b_{i}=1}=\int_{0}^{\infty} d \tau G\left(\left(\hat{\mathbf{a}}_{i}-\gamma \hat{\mathbf{b}}_{i}\right) \tau\right)
$$

where again $\operatorname{Re}\left(\hat{\mathbf{a}}_{i}-\gamma \hat{\mathbf{b}}_{i}\right)>0$ has been assumed. Here and hereafter the carret designates the omission of the label such that

$$
\begin{aligned}
\hat{\mathbf{a}}_{i} & \equiv a_{1} a_{2} \cdots a_{i-1} a_{i+1} \cdots a_{N}=\prod_{j \neq i} a_{j} \\
\hat{\mathbf{a}}_{i_{1} i_{2}} & \equiv a_{1} \cdots a_{i_{1}-1} a_{i_{1}+1} \cdots a_{i_{2}-1} a_{i_{2}+1} \cdots a_{N}=\prod_{j \neq i_{1}, i_{2}} a_{j}, \\
\{\hat{a}\}_{i} & \equiv\{a\} \backslash a_{i} .
\end{aligned}
$$

The formula is then applied to the last line of (3.7) to give

$$
\begin{aligned}
& \left.\prod_{i=1}^{N} F\left(\kappa ;-\partial_{a_{i}}, \partial_{b_{i}}\right) \int_{0}^{\infty} d \tau G((\mathbf{a}-\gamma \mathbf{b}) \tau)\right|_{\{a\}=\{b\}=1} \\
= & \left.\prod_{i \neq j_{1}} F\left(\kappa ;-\partial_{a_{i}}, \partial_{b_{i}}\right) \int_{0}^{\infty} d \tau G\left(\left(\hat{\mathbf{a}}_{j_{1}}-\gamma \hat{\mathbf{b}}_{j_{1}}\right) \tau\right)\right|_{\{\hat{a}\}_{j_{1}}=\{\hat{b}\}_{j_{1}}=1} \quad\left(1 \leq{ }^{\forall} j_{1} \leq N\right),
\end{aligned}
$$

which is further reduced to

$$
=\left.\prod_{i \neq j_{1}, j_{2}} F\left(\kappa ;-\partial_{a_{i}}, \partial_{b_{i}}\right) \int_{0}^{\infty} d \tau G\left(\left(\hat{\mathbf{a}}_{j_{1} j_{2}}-\gamma \hat{\mathbf{b}}_{j_{1} j_{2}}\right) \tau\right)\right|_{\{\hat{a}\}_{j_{1} j_{2}}=\{\hat{b}\}_{j_{1} j_{2}}=1} .
$$

Repeating these steps, we finally obtain the relation in which only one $a$ and $b$ survive to find,

$$
\begin{aligned}
& =\left.F\left(\kappa ;-\partial_{\hat{a}_{j_{1} \cdots j_{N-1}}}, \partial_{\hat{b}_{j_{1} \cdots j_{N-1}}}\right) \int_{0}^{\infty} d \tau G((\mathbf{a}-\gamma \mathbf{b}) \tau)\right|_{\hat{a}_{j_{1} \cdots j_{N-1}}=\hat{b}_{j_{1} \cdots j_{N-1}}=1} \\
& =\left.F\left(\kappa ;-\partial_{a}, \partial_{b}\right) \int_{0}^{\infty} d \tau G((a-\gamma b) \tau)\right|_{a=b=1} \\
& =\frac{1}{1-\gamma}
\end{aligned}
$$

which proves the desired relation (3.6) in view of (3.10). Thus (3.6) has been confirmed so that $(3.5)$ is found to be

$$
\begin{aligned}
& Z_{N}=\frac{e^{i h J T}}{1-e^{-i h T}}+\frac{e^{-i h J T}}{1-e^{i h T}}=\frac{\sin ((J+1 / 2) h T)}{\sin (h T / 2)}: S U(2), \\
& Z_{N}=\frac{e^{-i h K T}}{1-e^{-i h T}} \quad: \quad S U(1,1) .
\end{aligned}
$$


The conclusion is therefore that the WKB is exact under coherent state path integrals for $S U(2)$ and $S U(1,1)$ (in the discrete series).

Now we proceed to establish the formula (3.11): write

$$
\begin{aligned}
F\left(\kappa ;-\partial_{a_{i}}, \partial_{b_{i}}\right) & \left.\int_{0}^{\infty} d \tau G((\mathbf{a}-\gamma \mathbf{b}) \tau)\right|_{a_{i}=b_{i}=1} \\
& =\int_{0}^{\infty} d \tau F\left(\kappa ; \hat{\mathbf{a}}_{i} \tau, \gamma \hat{\mathbf{b}}_{i} \tau\right) G\left(\left(\hat{\mathbf{a}}_{i}-\gamma \hat{\mathbf{b}}_{i}\right) \tau\right)
\end{aligned}
$$

so that the formula becomes

$$
\int_{0}^{\infty} d \tau F(\kappa ; a \tau, \gamma b \tau) G((a-\gamma b) \tau)=\int_{0}^{\infty} d \tau G((a-\gamma b) \tau)
$$

To make clear the $\kappa$-dependence of $F$, introduce

$$
F(\kappa ; x, y)=\sum_{n=0}^{\infty} \frac{\kappa^{n}}{n !} F_{n}(x, y)
$$

First let us prove

$$
\int_{0}^{\infty} d \tau F_{n}(\tau, c \tau) G((1-c) \tau)=\frac{\delta_{n, 0}}{1-c}, \operatorname{Re}(1-c)>0
$$

$F_{n}$ is written explicitly as

$$
\begin{aligned}
F_{n}(\tau, c \tau) & =\frac{n !}{2 \pi i} \oint_{C_{0}} \frac{d z}{z^{n+1}} \exp \left[\sum_{m=1}^{\infty} \frac{(-)^{m}}{m}(\tau z)^{m}\left\{\left(1+c^{m}\right)-\frac{m}{m+1} \tau\left(1-c^{m+1}\right)\right\}\right] \\
& =\frac{n !}{2 \pi i} \oint_{C_{0}} \frac{d z}{z^{n+1}} \exp \left\{-\ln (1+\tau z)(1+c \tau z)-\frac{1}{z} \ln \left(\frac{1+\tau z}{1+c \tau z}\right)+(1-c) \tau\right\}
\end{aligned}
$$

where the contour $C_{0}$ is supposed to enclose the origin with sufficiently small radius. Then (3.20) becomes

$$
\begin{aligned}
\text { L.H.S.of }(3.20) & \equiv \lim _{R \rightarrow \infty} \Omega_{n}(R) \\
\qquad \Omega_{n}(R) & =\frac{n !}{2 \pi i} \oint_{C_{R}} \frac{d z}{z^{n+1}} \int_{0}^{R} \frac{d \tau}{(1+\tau z)^{2}} \exp \left\{-\left(\frac{1}{z}-1\right) \ln \left(\frac{1+\tau z}{1+c \tau z}\right)\right\},
\end{aligned}
$$

where $R$, permitting us to sum up the series to the logarithm in (3.21), is a cut-off and $C_{R} ;|z R|<1$ which encloses the origin. A change of variable

$$
\tau \mapsto \rho=\ln \left(\frac{1+\tau z}{1+c \tau z}\right), \quad \frac{d \tau}{(1+\tau z)^{2}}=\frac{d \rho}{(1-c) z} e^{-\rho}
$$


brings us to

$$
\begin{aligned}
\Omega_{n}(R) & =\frac{n !}{2 \pi i} \oint_{C_{R}} \frac{d z}{z^{n+1}} \int_{0}^{\rho(R)} \frac{d \rho}{(1-c) z} \exp \left(-\frac{\rho}{z}\right) \\
& =\frac{1}{1-c}\left(\delta_{n, 0}-\Psi_{n}(R)\right),
\end{aligned}
$$

where

$$
\begin{aligned}
\Psi_{n}(R) & \equiv \frac{n !}{2 \pi i} \oint_{C_{R}} \frac{d z}{z^{n+1}} \exp \left\{-\frac{1}{z} \ln \left(\frac{1+R z}{1+c R z}\right)\right\} \\
& =\left.\left(\frac{d}{d z}\right)^{n} \exp \left\{-\frac{1}{z} \ln \left(\frac{1+R z}{1+c R z}\right)\right\}\right|_{z=0},
\end{aligned}
$$

$\Psi_{n}(R)$ satisfies a relation as;

$$
\begin{aligned}
& \Psi_{n}(R)=\sum_{r=1}^{n}\left(\begin{array}{c}
n-1 \\
r-1
\end{array}\right) \psi_{r}(R) \Psi_{n-r}(R), \quad \Psi_{0}(R)=e^{-(1-c) R}, \\
& \psi_{n}(R) \equiv \frac{(-)^{n}}{n+1} n ! R^{n+1}\left(1-c^{n+1}\right) .
\end{aligned}
$$

Apparently $\Psi_{n}(R)$ is found to be

$$
\Psi_{n}(R)=(\text { polynomial of } R \text { of order } 2 n) \times e^{-(1-c) R}
$$

and consequently

$$
\lim _{R \rightarrow \infty} \Psi_{n}(R)=0 \quad(n<\infty)
$$

This completes the proof of (3.20). Then with setting $\tau \mapsto a \tau ; c=\gamma b / a$, we see (3.18) holds. Thus we have proven the formula (3.11).

\section{DISCUSSION}

The discussion in the previous section leads us to the fact that in the path integral representation, (2.5) and (2.27), the WKB approximation is exact, whose result, as can been seen such as from (3.15), is independent of the number of time $\operatorname{slices}(N)$. Therefore putting $N \rightarrow \infty$ the result may be regarded as an infinite dimensional version of $\mathrm{D}-\mathrm{H}$ 
theorem. To see the meaning of this result clearly, let us re-examine the character formula (2.17). First rewrite it with keeping the exponent always correct up to $O(\epsilon)$ as

$$
Z(T)=\lim _{N \rightarrow \infty} e^{i h J T} \prod_{j=1}^{N} \int_{\mathrm{PBC}} d \mu\left(\xi_{j}^{*}, \xi_{j}\right) \exp \left\{2 J \ln \left(\frac{1+e^{-i \epsilon h} \xi_{j}^{*} \xi_{j-1}}{1+\xi_{j}^{*} \xi_{j}}\right)\right\},
$$

which becomes, by making use of local $U(1)$ invariance of the measure under

$$
\xi_{j} \mapsto \xi_{j} e^{-i j \epsilon h}
$$

to

$$
\begin{aligned}
Z(T) & =\left.\lim _{N \rightarrow \infty} e^{i h J T} \prod_{j=1}^{N} \int d \mu\left(\xi_{j}^{*}, \xi_{j}\right) \exp \left\{2 J \ln \left(\frac{1+\xi_{j}^{*} \xi_{j-1}}{1+\xi_{j}^{*} \xi_{j}}\right)\right\}\right|_{\xi_{0}=\xi_{N} e^{-i h T}} \\
& =\left.\lim _{N \rightarrow \infty} e^{i h J T} \prod_{j=1}^{N} \int d \mu\left(\xi_{j}^{*}, \xi_{j}\right)\left\langle\xi_{j} \mid \xi_{j-1}\right\rangle\right|_{\xi_{0}=\xi_{N} e^{-i h T}} \\
& =e^{i h J T} \int d \mu\left(\xi^{*}, \xi\right)\left\langle\xi \mid \xi e^{-i h T}\right\rangle,
\end{aligned}
$$

where we have used (2.6) from the first to the second line and the resolution of unity (2.7) from the second to the third line. After carrying out the trivial integration with respect to the phase of $\xi$, (4.3) reduces to

$$
\begin{aligned}
Z(T) & =e^{i h J T}(2 J+1) \int_{0}^{\infty} \frac{d u}{(1+u)^{2}}\left(\frac{1+e^{-i h T} u}{1+u}\right)^{2 J} \\
& =e^{i h J T} \sum_{n=0}^{2 J} e^{-i n h T}=\frac{\sin ((J+1 / 2) h T)}{\sin (h T / 2)} .
\end{aligned}
$$

Let us take another point of view: make a change of variable

$$
u \quad \mapsto \quad z=-\ln \left(\frac{1+e^{-i h T} u}{1+u}\right),
$$

which brings the first line of (4.4) to

$$
Z(T)=e^{i h J T}(2 J+1) \int_{C} \frac{d z}{1-e^{-i h T}} e^{-(2 J+1) z} .
$$

Since the integrand in (4.6) has no singularity on $z$-plane, the original contour $C$ can be deformed arbitrarily. The new contour $C_{+}+C_{R}+C_{-}$illustrated in Fig. 11 provides an interesting view point about the property of the character formula; which reads 


$$
\begin{aligned}
Z(T) & =\frac{e^{i h J T}}{1-e^{-i h T}}\left\{\int_{0}^{\infty} d x e^{-x}-\int_{0}^{\infty} d x e^{-x-i(2 J+1) h T}\right\} \\
& =\left(\frac{e^{i h J T}}{1-e^{-i h T}}+\frac{e^{-i h J T}}{1-e^{i h T}}\right) \int_{0}^{\infty} d x e^{-x},
\end{aligned}
$$

giving the relation

$$
\begin{aligned}
Z(T) & =\frac{\sin ((J+1 / 2) h T)}{\sin (h T / 2)}=\frac{e^{i h J T}}{1-e^{-i h T}}+\frac{e^{-i h J T}}{1-e^{i h T}} \\
& =\operatorname{Tr} e^{-i h\left(a^{\dagger} a-J\right) T}+\operatorname{Tr} e^{i h\left(a^{\dagger} a-J\right) T} .
\end{aligned}
$$

Hence it is now clear that the character formula can be expressed as the sum of partition functions of two harmonic oscillators with frequency $\pm h$.

The situation is almost the same in $S U(1,1)$ case: corresponding to (4.1), we have

$$
Z(T)=\lim _{N \rightarrow \infty} e^{-i h K T} \prod_{j=1}^{N} \int_{\mathrm{PBC}} d \mu\left(\xi_{j}^{*}, \xi_{j}\right) \exp \left\{-2 K \ln \left(\frac{1-e^{-i \epsilon h} \xi_{j}^{*} \xi_{j-1}}{1-\xi_{j}^{*} \xi_{j}}\right)\right\}
$$

By following the same procedure from (4.3) to (4.4), $Z(T)$ can be calculated to be

$$
\begin{aligned}
Z(T) & =e^{-i h K T} \int d \mu\left(\xi^{*}, \xi\right)\left\langle\xi \mid \xi e^{-i h T}\right\rangle \\
& =e^{-i h K T}(2 K-1) \int_{0}^{1} \frac{d u}{(1-u)^{2}}\left(\frac{1-u}{1-e^{-i h T} u}\right)^{2 K} \\
& =e^{-i h K T} \sum_{n=0}^{\infty} e^{-i n h T}=\frac{e^{-i h K T}}{1-e^{-i h T}}
\end{aligned}
$$

Also similar from (4.5) to (4.8), the final result (4.10) can be interpreted as follows: the change of variable,

$$
u \quad \mapsto \quad z=-\ln \left(\frac{1-u}{1-e^{-i h T} u}\right)
$$

gives us

$$
Z(T)=e^{-i h K T}(2 K-1) \int_{C} \frac{d z}{1-e^{-i h T}} e^{-(2 K-1) z}
$$

It is easily recognized from Fig. 2 that there exists only one line integral which can be deformed to the line $0 \leq x<\infty$, corresponding to the fact that there is only one harmonic oscillator; 


$$
Z(T)=\frac{e^{-i h K T}}{1-e^{-i h T}}=\operatorname{Tr} e^{-i h\left(a^{\dagger} a+K\right)}
$$

The difference between contours in Fig. 1 and Fig. 2 is direct consequence of the difference between compact and non-compact phase spaces. This point of view will be also developed and become more transparent in the appendices.

Therefore we can see the reason why the WKB is exact; however the situation is not always the case even in $S U(2)$ as was mentioned in the introduction; if "the periodic coherent states [2]',

$$
\begin{aligned}
|\phi\rangle & \equiv \frac{1}{\sqrt{2 \pi}} \sum_{M=-J}^{J} e^{i(M+\Delta) \phi}|J, M\rangle, \quad-\pi \leq \phi<\pi, J=0,1 / 2,1,3 / 2, \cdots, \\
\Delta & =J-[J], \quad[x]=\text { integer part of } x
\end{aligned}
$$

is adopted then the final exponent is the Nielsen-Rohrlich form [1];

$$
\begin{aligned}
Z(T)= & \operatorname{Tr} e^{-i H T}=\lim _{N \rightarrow \infty} \sum_{n=-\infty}^{\infty} \int_{-\pi+2 n \pi}^{\pi+2 n \pi} d \phi_{N} \prod_{k=1}^{N-1} \int_{-\infty}^{\infty} d \phi_{k} \\
& \times\left.\int_{0}^{\pi} \lambda \sin \theta_{k} d \theta_{k} \exp \left\{i\left(\lambda \cos \theta_{k}+\Delta\right)\left(\phi_{k}-\phi_{k-1}\right)-i \epsilon h \lambda \cos \theta_{k}\right\}\right|_{\phi_{0}=\phi_{N}+2 \pi n}
\end{aligned}
$$

where $\lambda=J+1 / 2$. Naive continuum limit of (4.15) matches with that of (2.17) by putting

$$
\xi=e^{i \phi} \tan \frac{\theta}{2}
$$

The classical equations of motion, in terms of variables $\phi_{k}$ and $p_{k} \equiv \lambda \cos \theta_{k}$, are given by

$$
\dot{\phi}(t)=h, \quad \dot{p}(t)=0 .
$$

Apparently there is no solution compatible to the boundary condition, $\phi(0)=\phi(T)+2 n \pi$. However in terms of $\phi_{k}$ and $\theta_{k}$,

$$
\sin \theta(t)(\dot{\phi}(t)-h)=0, \quad \sin \theta(t) \dot{\theta}(t)=0
$$

There are solutions,

$$
\theta=0 \text { or } \pi
$$


In view of $(4.16), \theta=0(\pi)$ corresponds to $\xi_{c}=0(\infty)$ respectively, which tempts us build up the relationship between our formula (2.17) and (4.15). The task is now undertaken [12.

The final comment is on the difference between (1.5) and (1.6); namely if we had used the continuum path integral formula in (3.6), we would get $\sin (J T) / \sin (T / 2)$ instead of

the correct one; $\sin ((J+1 / 2)) T / \sin (T / 2)$. The models which we have been considering are very much alike to (three dimensional) Chern-Simons theory [13]. So the issue that $J \rightarrow J+1 / 2$ (Weyl shift) may correspond to the Coxeter shift, $k \rightarrow k+2$ (where $k$ denotes a level) in the Chern-Simons case. Thus if it would be possible to perform the integration in discretized version of the Chern-Simons theory, we could get the correct value $k+2$, which will be an interesting subject in the future.

Another task for us is such that owing to the technique in the appendix of obtaining the generalized coherent states from the canonical coherent one, we could generalize our discussion to the case of Grassmannian manifold.

\section{APPENDIX A: COHERENT STATES FROM A GEOMETRICAL VIEW POINT}

We first summarize the mathematical description (tensor product method [6]) of constructing coherent state for $S U(2)(S U(1,1))$ as an example of compact (non-compact) case in this appendix $\mathbf{A}$.

\section{1. $S U(2)$ case}

By parameterizing a point of $\mathbf{C} P^{1}=\left\{P \in M(2, \mathbf{C}) \mid \operatorname{tr} P=1, \quad P^{\dagger}=P, \quad P^{2}=P\right\}$ as

$$
\begin{aligned}
P & =\frac{1}{1+|\xi|^{2}}\left(\begin{array}{cc}
1 & \xi^{*} \\
\xi & |\xi|^{2}
\end{array}\right)=\hat{u}(\xi) \hat{u}^{\dagger}(\xi), \quad \hat{u}(\xi)=\frac{u(\xi)}{|u(\xi)|} \\
u(\xi) & =\left(\begin{array}{l}
1 \\
\xi
\end{array}\right)=\mathbf{e}_{0}+\xi \mathbf{e}_{1} \in \mathbf{C}^{2}-\{0\} \quad,
\end{aligned}
$$

$S U(2)$ system is described as a Hamiltonian system with symplectic structure 


$$
\begin{aligned}
\omega & =i \operatorname{tr}(P d P \wedge d P) \\
& =i d \hat{u}^{\dagger}(\xi)\left(1-\hat{u}(\xi) \hat{u}^{\dagger}(\xi)\right) \wedge d \hat{u}(\xi) \\
& =i \frac{d \xi^{*} \wedge d \xi}{\left(1+|\xi|^{2}\right)^{2}}
\end{aligned}
$$

The vectors $\mathbf{e}_{0}, \mathbf{e}_{1}$ in (A1) are basis vectors of $\mathbf{C}^{2}$ being 2-dimensional representation space of $S U(2)$. Our convention on this basis is

$$
\begin{aligned}
& d \rho_{1 / 2}\left(J_{3}\right) \mathbf{e}_{m}=(-1 / 2+m) \mathbf{e}_{m} \\
& d \rho_{1 / 2}\left(J_{+}\right) \mathbf{e}_{m}=\sqrt{(m+1)(1-m)} \mathbf{e}_{m+1}, \quad(m=0,1) \\
& d \rho_{1 / 2}\left(J_{-}\right) \mathbf{e}_{m}=\sqrt{m(2-m)} \mathbf{e}_{m-1} .
\end{aligned}
$$

The dynamical variables are elements of $s u(2)$ being realized as functions on $\mathbf{C} P^{1}$ by a map

$$
\begin{aligned}
s u(2) \ni X \mapsto F_{X}(P) & =\operatorname{tr}\left(P d \rho_{1 / 2}(X)\right)=\operatorname{tr}\left(\hat{u}(\xi) \hat{u}^{\dagger}(\xi) d \rho_{1 / 2}(X)\right) \\
& =\hat{u}^{\dagger}(\xi) d \rho_{1 / 2}(X) \hat{u}(\xi) \in \mathbf{R} .
\end{aligned}
$$

The Poisson bracket between two variables $X, Y \in s u(2)$ is defined in terms of their corresponding vector fields $V_{X}, V_{Y}$ and $\omega^{-1}$ by

$$
\left\{F_{X}, F_{Y}\right\}_{\text {P.B. }} \equiv \omega^{-1}\left(V_{X}, V_{Y}\right)
$$

Taking $J_{3}$ as a Hamiltonian, we find that the classical action is given by

$$
S=\frac{1}{2} \int_{0}^{T} d t\left(i \frac{\xi^{*} \dot{\xi}-\dot{\xi}^{*} \xi}{1+|\xi|^{2}}+\frac{1-|\xi|^{2}}{1+|\xi|^{2}}\right)
$$

There are various symplectic structures corresponding to higher spin representation. They are given by an embedding

$$
\mathbf{C} P^{1} \rightarrow \mathbf{C} P^{2 J}=\left\{P \in M(2 J+1, \mathbf{C}) \mid \operatorname{tr} P=1, P^{\dagger}=P, P^{2}=P\right\}
$$

To see this explicitly, we first note the well known fact that the symmetric sector of the tensor product $\otimes^{2 J} \mathbf{C}^{2}$ is invariant under the action of $\left(\otimes^{2 J} \rho_{1 / 2}\right)(g), g \in G L(2, \mathbf{C})$. In order to pick up this subspace, $V_{J}$, define a generating function of basis vectors of $V_{J}$ : 


$$
\begin{aligned}
V_{J} \ni \mathcal{G}_{J}(t) & =\rho_{J}\left(e^{t J_{+}}\right)\left(\mathbf{e}_{0} \otimes \cdots \otimes \mathbf{e}_{0}\right) \\
& =\sum_{m=0}^{2 J} t^{m} \sum_{|\mathbf{n}|=m} \mathbf{e}_{n_{1}} \otimes \mathbf{e}_{n_{2}} \otimes \cdots \otimes \mathbf{e}_{n_{2 J}} \\
|\mathbf{n}| & =\sum_{i=1}^{2 J} n_{i}, \quad n_{i}=0,1
\end{aligned}
$$

where

$$
\rho_{J}(g) v \equiv\left(\otimes^{2 J} \rho_{1 / 2}\right)(g) v, \quad g \in G L(2, \mathbf{C}), v \in V_{J}
$$

Then we can find a set of basis vectors for $V_{J}$ :

$$
\begin{aligned}
\mathbf{e}_{m}^{(J)}= & \left.\left(\begin{array}{c}
2 J \\
m
\end{array}\right)^{-1 / 2} \frac{1}{m !}\left(\frac{d}{d t}\right)^{m}\right|_{t=0} \mathcal{G}_{J}(t)(0 \leq m \leq 2 J) \\
= & \left(\begin{array}{c}
2 J \\
m
\end{array}\right)^{-1 / 2} \sum_{|\mathbf{n}|=m} \mathbf{e}_{n_{1}} \otimes \mathbf{e}_{n_{2}} \otimes \cdots \otimes \mathbf{e}_{n_{2 J}}, \\
& \left(\mathbf{e}_{m}^{(J)}, \mathbf{e}_{n}^{(J)}\right)=\delta_{m, n} .
\end{aligned}
$$

The spin- $J$ representation of $s u(2)$ on $V_{J}$ is expressed, by putting $g=e^{t X}, X \in s u(2)$ in (A9), as

$$
d \rho_{J}(X)=\sum_{k=1}^{2 J} \overbrace{\rho_{1 / 2}(1) \otimes \cdots \otimes \rho_{1 / 2}(1)}^{k-1} \otimes d \rho_{1 / 2}(X) \otimes \overbrace{\rho_{1 / 2}(1) \otimes \cdots \otimes \rho_{1 / 2}(1)}^{2 J-k} .
$$

In particular, corresponding to (2.2), we have

$$
\begin{aligned}
d \rho_{J}\left(J_{3}\right) \mathbf{e}_{m}^{(J)} & =(-J+m) \mathbf{e}_{m}^{(J)}, \\
d \rho_{J}\left(J_{+}\right) \mathbf{e}_{m}^{(J)} & =\sqrt{(m+1)(2 J-m)} \mathbf{e}_{m+1}^{(J)}, \quad(0 \leq m \leq 2 J) \\
d \rho_{J}\left(J_{-}\right) \mathbf{e}_{m}^{(J)} & =\sqrt{m(2 J-m+1)} \mathbf{e}_{m-1}^{(J)} .
\end{aligned}
$$

Now defining

$$
\begin{aligned}
u(\xi) & \mapsto u_{J}(\xi)=\mathcal{G}_{J}(\xi) \\
& =\sum_{m=0}^{2 J}\left(\begin{array}{c}
2 J \\
m
\end{array}\right)^{1 / 2} \xi^{m} \mathbf{e}_{m}^{(J)} \in \mathbf{C}^{2 J+1}-\{0\}, \quad \hat{u}_{J}(\xi)=\frac{u_{J}(\xi)}{\left|u_{J}(\xi)\right|}
\end{aligned}
$$

we find an explicit parameterization of the embedding (A7): 


$$
P_{J}=\hat{u}_{J}(\xi) \hat{u}_{J}^{\dagger}(\xi) \in \mathbf{C} P^{2 J}
$$

Again the dynamical variables are given by

$$
F_{X}^{(J)}(P)=\operatorname{tr}\left(P_{J} d \rho_{J}(X)\right)=\hat{u}_{J}^{\dagger}(\xi) d \rho_{J}(X) \hat{u}_{J}(\xi)
$$

with Poisson brackets being defined with respect to the symplectic form

$$
\begin{aligned}
\omega_{J} & =i \operatorname{tr}\left(P_{J} d P_{J} \wedge d P_{J}\right) \\
& =i d \hat{u}_{J}^{\dagger}(\xi)\left(1-\hat{u}_{J}(\xi) \hat{u}_{J}^{\dagger}(\xi)\right) \wedge d \hat{u}_{J}(\xi) \\
& =2 i J \frac{d \xi^{*} \wedge d \xi}{\left(1+|\xi|^{2}\right)^{2}}
\end{aligned}
$$

The classical action is read as

$$
S_{J}=J \int_{0}^{T} d t\left(i \frac{\xi^{*} \dot{\xi}-\dot{\xi}^{*} \xi}{1+|\xi|^{2}}+\frac{1-|\xi|^{2}}{1+|\xi|^{2}}\right)
$$

Thus one might expect the path integral expression

$$
\int \prod_{0 \leq t \leq T} \omega_{J}(t) \exp \left(i J \int_{0}^{T} d t\left\{i \frac{\xi^{*} \dot{\xi}-\dot{\xi}^{*} \xi}{1+|\xi|^{2}}+\frac{1-|\xi|^{2}}{1+|\xi|^{2}}\right\}\right)
$$

could correspond to the trace formula $\operatorname{Tr}\left(e^{-i J_{3} T}\right)$ with a suitable boundary condition. Such an expectation is, however, too naive and (A18) lacks much information as was mentioned in the introduction. As a final comment, it should be noted that the vector space $V_{J}$ spanned by $\left\{\mathbf{e}_{m}^{(J)} ; 0 \leq m \leq 2 J\right\}$ is just the $2 J+1$ dimensional representation space of $S U(2)$ and a coherent state in this representation, $u_{J}(\xi)$, given by a symmetric tensor product, is nothing but a generating function of the basis vectors of $V_{J}$.

\section{2. $S U(1,1)$ case}

The phase space $D_{(1,1)}$ for $S U(1,1)$ system is given by

$$
\begin{aligned}
D_{(1,1)} & \equiv\left\{\left.\xi \in \mathbf{C}|| \xi\right|^{2}<1\right\} \\
& =\left\{P \in M(2, \mathbf{C}) \mid \operatorname{tr} P=1, \eta P^{\dagger} \eta=P, P^{2}=P\right\},
\end{aligned}
$$


where $\eta=\operatorname{diag}(1,-1)$. An explicit form of $P$ is

$$
\begin{aligned}
P & =v(\xi)(\eta v(\xi))^{\dagger}=v(\xi) v(\xi)^{\dagger} \eta \\
& =\frac{1}{1-|\xi|^{2}}\left(\begin{array}{cc}
1 & -\xi^{*} \\
\xi & -|\xi|^{2}
\end{array}\right),
\end{aligned}
$$

with

$$
v(\xi)=\frac{1}{\sqrt{1-|\xi|^{2}}}\left(\begin{array}{l}
1 \\
\xi
\end{array}\right), \quad v(\xi)^{\dagger} \eta v(\xi)=1
$$

The symplectic form on this phase space is found, as (A2), to be

$$
\omega=i \operatorname{tr}(P d P \wedge d P)=-i \frac{d \xi^{*} \wedge d \xi}{\left(1-|\xi|^{2}\right)^{2}}
$$

Again similar to (A4) the map

$$
s u(1,1) \ni X \mapsto F_{X}(P)=\operatorname{tr}(P X)=v(\xi)^{\dagger} \eta X v(\xi)
$$

gives us dynamical variables and classical mechanics on this phase space are described with symplectic form (A22). Various symplectic structures are obtained by a similar recipe as above. However they do not provide unitary representation of $S U(1,1)$ due to the indefinite metric $\eta$.

To obtain the discrete series of unitary representation of $S U(1,1)$ as well as coherent states, an embedding $D_{(1,1)} \rightarrow \mathbf{C} P^{\infty}$ is needed: first construct the fundamental representation (limit of discrete series) as

$$
\begin{aligned}
d \tilde{\rho}_{1 / 2}\left(K_{3}\right) & =\sum_{n=0}^{\infty}(n+1 / 2) \mathbf{e}_{n} \mathbf{e}_{n}^{T} \\
d \tilde{\rho}_{1 / 2}\left(K_{+}\right) & =\sum_{n=0}^{\infty}(n+1) \mathbf{e}_{n+1} \mathbf{e}_{n}^{T} \\
d \tilde{\rho}_{1 / 2}\left(K_{-}\right) & =-\sum_{n=0}^{\infty}(n+1) \mathbf{e}_{n} \mathbf{e}_{n+1}^{T} \\
\left(\mathbf{e}_{m}, \mathbf{e}_{n}\right) & =\mathbf{e}_{m}^{T} \mathbf{e}_{n}=\delta_{m, n}
\end{aligned}
$$

and the coherent state; by a map 


$$
D_{(1,1)} \ni \xi \mapsto u(\xi)=\sum_{n=0}^{\infty} \xi^{n} \mathbf{e}_{n} \in l^{2}(\mathbf{C})-\{0\} \quad, \hat{u}(\xi)=\frac{u(\xi)}{|u(\xi)|}
$$

Here $\tilde{\rho}_{1 / 2}$ is

$$
\tilde{\rho}_{1 / 2}: S L(2, \mathbf{C}) \ni g \mapsto \tilde{\rho}_{1 / 2}(g) \in G L\left(l^{2}(\mathbf{C})\right)
$$

and $\mathbf{e}_{n}$ 's are basis vectors of $l^{2}(\mathbf{C})$. Formal definition of $e^{t K_{+}}$is given by

$$
\tilde{\rho}_{1 / 2}\left(e^{t K_{+}}\right)=\sum_{m=0}^{\infty} \sum_{n=0}^{\infty} t^{m}\left(\begin{array}{c}
m+n \\
m
\end{array}\right) \mathbf{e}_{n+m} \mathbf{e}_{n}^{T},
$$

which generates a coherent state, when acting on $\mathbf{e}_{0}$ :

$$
\tilde{\rho}_{1 / 2}\left(e^{t K_{+}}\right) \mathbf{e}_{0}=u(t),|t|<1
$$

The resolution of unity is written as

$$
\int_{D_{(1,1)}} d \mu(\xi) \hat{u}(\xi) \hat{u}(\xi)^{\dagger}=\tilde{\rho}_{1 / 2}(1)=\sum_{n=0}^{\infty} \mathbf{e}_{n} \mathbf{e}_{n}^{T}
$$

where we have introduced a regularized measure $d \mu(\xi)$,

$$
d \mu(\xi)=\lim _{\epsilon \rightarrow+0} \frac{\epsilon}{\pi} \frac{d \xi^{*} d \xi}{\left(1-|\xi|^{2}\right)^{2-\epsilon}}
$$

The remainder of the discrete series are obtained by

$$
D_{(1,1)} \ni \xi \rightarrow P_{K}=\hat{u}_{K}(\xi) \hat{u}_{K}^{\dagger}(\xi) \in \mathbf{C} P^{\infty}
$$

where $\hat{u}_{K}(\xi)$ is given by the same procedure in the previous section: first, define a generating function of basis vectors for symmetric sector $\left(\tilde{V}_{K}\right)$ of $\otimes^{2 K} l^{2}(\mathbf{C})$

$$
\begin{aligned}
\tilde{V}_{K} \ni \tilde{\mathcal{G}}_{K}(t) & =\tilde{\rho}_{K}\left(e^{t K_{+}}\right)\left(\mathbf{e}_{0} \otimes \cdots \otimes \mathbf{e}_{0}\right)(|t|<1) \\
& =\sum_{m=0}^{\infty} t^{m} \sum_{|\mathbf{n}|=m} \mathbf{e}_{n_{1}} \otimes \mathbf{e}_{n_{2}} \otimes \cdots \otimes \mathbf{e}_{n_{2 K}}, \\
|\mathbf{n}| & =\sum_{i=1}^{2 K} n_{i}, \quad n_{i}=0,1,2, \cdots,
\end{aligned}
$$

where 


$$
\tilde{\rho}_{K}(g) \tilde{v}=\left(\otimes^{2 K} \tilde{\rho}_{1 / 2}\right)(g) \tilde{v} \quad g \in S L(2, \mathbf{C}), \tilde{v} \in \tilde{V}_{K}
$$

With a similar manner as above a set of basis vectors for $\tilde{V}_{K}$;

$$
\begin{aligned}
\mathbf{e}_{m}^{(K)}= & \left.\left(\begin{array}{c}
2 K+m-1 \\
m
\end{array}\right)^{-1 / 2} \frac{1}{m !}\left(\frac{d}{d t}\right)^{m}\right|_{t=0} \tilde{\mathcal{G}}_{K}(t)(m=0,1,2, \cdots) \\
= & \left(\begin{array}{c}
2 K+m-1 \\
m
\end{array}\right)^{-1 / 2} \sum_{|\mathbf{n}|=m} \mathbf{e}_{n_{1}} \otimes \mathbf{e}_{n_{2}} \otimes \cdots \otimes \mathbf{e}_{n_{2 K}}, \\
& \left(\mathbf{e}_{m}^{(K)}, \mathbf{e}_{n}^{(K)}\right)=\delta_{m, n}
\end{aligned}
$$

is found. Thus "spin"- $K$ representation of $X \in s u(1,1)$ is expressed as

$$
d \tilde{\rho}_{K}(X)=\sum_{k=1}^{2 K} \overbrace{\tilde{\rho}_{1 / 2}(1) \otimes \cdots \otimes \tilde{\rho}_{1 / 2}(1)}^{k-1} \otimes d \tilde{\rho}_{1 / 2}(X) \otimes \overbrace{\tilde{\rho}_{1 / 2}(1) \otimes \cdots \otimes \tilde{\rho}_{1 / 2}(1)}^{2 K-k},
$$

yielding (corresponding to 2.23$)$ )

$$
\begin{aligned}
d \tilde{\rho}_{K}\left(K_{3}\right) \mathbf{e}_{m}^{(K)} & =(K+m) \mathbf{e}_{m}^{(K)}, \\
d \tilde{\rho}_{K}\left(K_{+}\right) \mathbf{e}_{m}^{(K)} & =\sqrt{(m+1)(2 K+m)} \mathbf{e}_{m+1}^{(K)}, \\
d \tilde{\rho}_{K}\left(K_{-}\right) \mathbf{e}_{m}^{(K)} & =-\sqrt{m(2 K+m-1)} \mathbf{e}_{m-1}^{(K)} .
\end{aligned}
$$

We now define $\hat{u}_{K}(\xi)$ as

$$
\begin{aligned}
u_{K}(\xi) & =\tilde{\mathcal{G}}_{K}(\xi) \\
& =\sum_{m=0}^{\infty}\left(\begin{array}{c}
2 K+m-1 \\
m
\end{array}\right){ }^{1 / 2} \xi^{m} \mathbf{e}_{m}^{(K)} \in l^{2}(\mathbf{C})-\{0\}, \quad \hat{u}_{K}(\xi)=\frac{u_{K}(\xi)}{\left|u_{K}(\xi)\right|},
\end{aligned}
$$

giving an explicit parameterization of $P_{K}$ in (A31). Integrating $P_{K}$ by use of the measure

$$
d \mu_{K}(\xi)=\frac{2 K-1}{\pi} \frac{d \xi^{*} d \xi}{\left(1-|\xi|^{2}\right)^{2}}
$$

we find the resolution of unity

$$
\int_{D(1,1)} d \mu_{K}(\xi) P_{K}=\tilde{\rho}_{K}(1)=\sum_{m=0}^{\infty} \mathbf{e}_{m}^{(K)} \mathbf{e}_{m}^{(K)^{T}}
$$

In view of (A32) and (A37), we again recognize that a coherent state in "spin"- $K$ representation is nothing but a generating function of basis vectors of the representation space $\tilde{V}_{K}$ 


\section{APPENDIX B: COHERENT STATES IN TERMS OF THE SCHWINGER BOSONS}

\section{1. $S U(2)$ case}

Following Schwinger [14], first consider a system of two bosonic oscillators:

$$
\left[a_{i}, a_{j}^{\dagger}\right]=\delta_{i, j}, \quad\left[a_{i}, a_{j}\right]=0, \quad\left[a_{i}^{\dagger}, a_{j}^{\dagger}\right]=0 \quad(i=1,2)
$$

and define operators

$$
\hat{J}_{3} \equiv \mathbf{a}^{\dagger} \frac{\sigma_{3}}{2} \mathbf{a}, \quad \hat{J}_{ \pm} \equiv \mathbf{a}^{\dagger} \frac{\sigma_{1} \pm i \sigma_{2}}{2} \mathbf{a}
$$

The Fock space is given as usual:

$$
\begin{gathered}
\left|\left(n_{1}, n_{2}\right)\right\rangle \equiv \frac{1}{\sqrt{n_{1} ! n_{2} !}}\left(a_{1}^{\dagger}\right)^{n_{1}}\left(a_{2}^{\dagger}\right)^{n_{2}}|(0,0)\rangle \quad\left(n_{i}=0,1,2 \cdots\right) \\
a_{i}|(0,0)\rangle=0(i=1,2)
\end{gathered}
$$

Introducing a constraint,

$$
\mathbf{a}^{\dagger} \mathbf{a}-2 J=0(J=0,1 / 2,1,3 / 2, \cdots)
$$

we can build up the spin- $J$ representation of $s u(2)$ out of the Fock space:

$$
\begin{aligned}
|(J+M, J-M)\rangle & =\frac{1}{\sqrt{(J+M) !(J-M) !}}\left(a_{1}^{\dagger}\right)^{J+M}\left(a_{2}^{\dagger}\right)^{J-M}|(0,0)\rangle \\
& \equiv|J, M\rangle .
\end{aligned}
$$

Thus the $S U(2)$ coherent state is identified with

$$
|\xi\rangle \equiv \frac{1}{\left(1+|\xi|^{2}\right)^{J}} \exp \left(\xi a_{1}^{\dagger} a_{2}\right)|(0,2 J)\rangle, \quad \xi \in \mathbf{C} .
$$

The constraint (B4) is realized by a projection operator in the Fock space 7 ;

\footnotetext{
${ }^{2}$ Rigorously speaking there need a regularization such that

$$
\mathbf{I}_{J}=\lim _{\varepsilon \rightarrow+0} \int_{0}^{2 \pi} \frac{d \lambda}{2 \pi} \exp \left\{i \lambda\left(\mathbf{a}^{\dagger} \mathbf{a}-2 J\right)-\varepsilon \mathbf{a}^{\dagger} \mathbf{a}\right\}
$$

in order to exchange the order of $\lambda$-integration and other operations in a safe manner. (See (B15)).
} 


$$
\mathbf{I}_{J} \equiv \int_{0}^{2 \pi} \frac{d \lambda}{2 \pi} \exp \left\{i \lambda\left(\mathbf{a}^{\dagger} \mathbf{a}-2 J\right)\right\}
$$

which is rewritten by use of canonical coherent state as

$$
\begin{aligned}
\mathbf{I}_{J} & =\int \frac{\left(d z^{*} d z\right)^{2}}{\pi^{2}} \frac{\left(d z^{*} d z^{\prime}\right)^{2}}{\pi^{2}}\left|\mathbf{z}^{\prime}\right\rangle\left\langle\mathbf{z}^{\prime}\left|\int_{0}^{2 \pi} \frac{d \lambda}{2 \pi} \exp \left\{i \lambda\left(\mathbf{a}^{\dagger} \mathbf{a}-2 J\right)\right\}\right| \mathbf{z}\right\rangle\langle\mathbf{z}| \\
& =\frac{1}{(2 J) !} \int \frac{\left(d z^{*} d z\right)^{2}}{\pi^{2}}|\mathbf{z}\rangle\langle(0,0)|\left(\mathbf{z}^{\dagger} \mathbf{a}\right)^{2 J} \exp \left(-\frac{1}{2} \mathbf{z}^{\dagger} \mathbf{z}\right)
\end{aligned}
$$

Making a change of variables

$$
\left(\begin{array}{c}
z_{1} \\
z_{2}
\end{array}\right)=\zeta\left(\begin{array}{l}
\xi \\
1
\end{array}\right), \quad\left(d z^{*} d z\right)^{2}=|\zeta|^{2} d \zeta^{*} d \zeta d \xi^{*} d \xi
$$

and integrating with respect to $\zeta$, we find

$$
\begin{aligned}
\mathbf{I}_{J} & =\frac{1}{(2 J) !} \int \frac{|\zeta|^{2} d \zeta^{*} d \zeta d \xi^{*} d \xi}{\pi^{2}} e^{\zeta\left(\xi a_{1}^{\dagger}+a_{2}^{\dagger}\right)}|(0,0)\rangle\langle(0,0)|\left\{\zeta^{*}\left(\xi^{*} a_{1}+a_{2}\right)\right\}^{2 J} e^{-|\zeta|^{2}\left(1+|\xi|^{2}\right)} \\
& =\frac{2 J+1}{\pi} \int \frac{d \xi^{*} d \xi}{\left(1+|\xi|^{2}\right)^{2}} \frac{\left(\xi a_{1}^{\dagger}+a_{2}^{\dagger}\right)^{2 J}}{\sqrt{(2 J) !}\left(1+|\xi|^{2}\right)^{J}}|(0,0)\rangle\langle(0,0)| \frac{\left(\xi^{*} a_{1}+a_{2}\right)^{2 J}}{\sqrt{(2 J) !}\left(1+|\xi|^{2}\right)^{J}} \\
& =\sum_{m, n=0}^{\infty} \frac{2 J+1}{\pi} \int \frac{d \xi^{*} d \xi}{\left(1+|\xi|^{2}\right)^{2}} \frac{1}{\left(1+|\xi|^{2}\right)^{2 J}} \frac{\left(\xi a_{1}^{\dagger} a_{2}\right)^{m}}{m !}|(0,2 J)\rangle\langle(0,2 J)| \frac{\left(\xi^{*} a_{1} a_{2}^{\dagger}\right)^{n}}{n !} \\
& =\frac{2 J+1}{\pi} \int \frac{d \xi^{*} d \xi}{\left(1+|\xi|^{2}\right)^{2}}|\xi\rangle\langle\xi| .
\end{aligned}
$$

Thus we see the equivalence between the projection operator $\mathbf{I}_{J}$ on the Fock space and the resolution of unity in the spin- $J$ representation of $S U(2)$.

There is another solution for constraint (B4)

$$
\left(\begin{array}{c}
z_{1} \\
z_{2}
\end{array}\right)=i \zeta\left(\begin{array}{l}
1 \\
\xi
\end{array}\right), \quad\left(d z^{*} d z\right)^{2}=|\zeta|^{2} d \zeta^{*} d \zeta d \xi^{*} d \xi
$$

which is related to the first solution $(\underline{B} 9)$ by an $S U(2)$ transformation

$$
\left(\begin{array}{c}
z_{1} \\
z_{2}
\end{array}\right) \mapsto\left(\begin{array}{cc}
0 & i \\
i & 0
\end{array}\right)\left(\begin{array}{c}
z_{1} \\
z_{2}
\end{array}\right)
$$

Upon this parameterization, we find another type of coherent states:

$$
|\xi\rangle\rangle \equiv \frac{1}{\left(1+|\xi|^{2}\right)^{J}} \exp \left(\xi a_{1} a_{2}^{\dagger}\right)|(2 J, 0)\rangle, \quad \xi \in \mathbf{C}
$$


which of course satisfy the resolution of unity

$$
\left.\mathbf{I}_{J}=\frac{2 J+1}{\pi} \int \frac{d \xi^{*} d \xi}{\left(1+|\xi|^{2}\right)^{2}}|\xi\rangle\right\rangle\langle\langle\xi|
$$

The transformation from $(\mathbb{B 1 0})$ to $(\mathbb{B 1 4})$ is achieved by $\xi \mapsto 1 / \xi$, which is nothing but the $S U(2)$ action on $\mathbf{C} P^{1}$ corresponding to the transformation (B12). It is now easy to see that the two critical points, $\xi=0, \infty$, found in section 2 are originated from the singular point, $\left(z_{1}, z_{2}\right)=0$, of two parameterizations (B9) and (B11).

If in (B7) the $\lambda$-integration is kept intact, we find another expression of the trace formula:

$$
\begin{aligned}
\operatorname{Tr} e^{-i J_{3} T} & =\int \frac{\left(d z^{*} d z\right)^{2}}{\pi^{2}}\left\langle\mathbf{z}\left|\int_{0}^{2 \pi} \frac{d \lambda}{2 \pi} \exp \left\{-i T \mathbf{a}^{\dagger}\left(\sigma_{3} / 2\right) \mathbf{a}+i \lambda\left(\mathbf{a}^{\dagger} \mathbf{a}-2 J\right)\right\}\right| \mathbf{z}\right\rangle \\
& =\lim _{\varepsilon \rightarrow+0} \int_{0}^{2 \pi} \frac{d \lambda}{2 \pi} e^{-2 i J \lambda} \int \frac{\left(d z^{*} d z\right)^{2}}{\pi^{2}}\left\langle\mathbf{z}\left|\exp \left\{-i T \mathbf{a}^{\dagger}\left(\sigma_{3} / 2\right) \mathbf{a}+(i \lambda-\varepsilon) \mathbf{a}^{\dagger} \mathbf{a}\right\}\right| \mathbf{z}\right\rangle \\
& =\lim _{\varepsilon \rightarrow+0} \int_{0}^{2 \pi} \frac{d \lambda}{2 \pi} e^{-2 i J \lambda} \frac{1}{\left(1-e^{i \lambda-\varepsilon-i T / 2}\right)\left(1-e^{i \lambda-\varepsilon+i T / 2}\right)}
\end{aligned}
$$

By putting $w=e^{-i \lambda}$, the desired form is found;

$$
\begin{aligned}
\operatorname{Tr} e^{-i J_{3} T} & =\lim _{\varepsilon \rightarrow+0} \oint_{|w|=1} \frac{d w}{2 \pi i} w^{2 J+1} \frac{1}{\left(w-e^{-\varepsilon-i T / 2}\right)\left(w-e^{-\varepsilon+i T / 2}\right)} \\
& =\frac{e^{-i(J+1 / 2) T}}{e^{-i T / 2}-e^{+i T / 2}}+\frac{e^{+i(J+1 / 2) T}}{e^{+i T / 2}-e^{-i T / 2}} \\
& =\frac{e^{-i J T}}{1-e^{+i T}}+\frac{e^{+i J T}}{1-e^{-i T}} .
\end{aligned}
$$

This result should be interpreted as follows: by deferring the $\lambda$-integration the Hamiltonian $(\operatorname{see}(\underline{B 15}))$ is regarded as a bilinear form of $\mathbf{a}^{\dagger}$ and $\mathbf{a}$. Thus under the canonical coherent state representation the path integral can be performed to yield a product of determinants as a function of $\lambda$. The remaining integration with respect to $\lambda$ can be expressed as the sum of the contributions from the singular points emerging from the reduction from $\mathbf{C}^{2}$ to $\mathbf{C} P^{1}$.

\section{2. $S U(1,1)$ case}

Introduce a set of operators

$$
\hat{K}_{3} \equiv \frac{1}{2}\left(a_{1}^{\dagger} a_{1}+a_{2}^{\dagger} a_{2}+1\right), \quad \hat{K}_{+} \equiv a_{1}^{\dagger} a_{2}^{\dagger}, \quad \hat{K}_{-} \equiv-a_{1} a_{2}
$$


and

$$
\hat{C} \equiv a_{2}^{\dagger} a_{2}-a_{1}^{\dagger} a_{1}
$$

The constraint

$$
\hat{C}-(2 K-1)=0(K=1 / 2,1,3 / 2, \cdots)
$$

to the Fock space gives us the $S U(1,1)$ "spin"- $K$ representation:

$$
\begin{aligned}
|(M, 2 K-1+M)\rangle & =\frac{1}{\sqrt{(2 K-1+M) ! M !}}\left(a_{1}^{\dagger}\right)^{M}\left(a_{2}^{\dagger}\right)^{2 K-1+M}|(0,0)\rangle \\
& \equiv|K, M\rangle \quad(M=0,1,2, \cdots) .
\end{aligned}
$$

Therefore the $S U(1,1)$ coherent state is given as

$$
|\xi\rangle \equiv\left(1-|\xi|^{2}\right)^{K} \exp \left(\xi a_{1}^{\dagger} a_{2}^{\dagger}\right)|(0,2 K-1)\rangle, \quad \xi \in D_{(1,1)}
$$

As in the case of $s u(2)$, the projection operator

$$
\mathbf{I}_{K} \equiv \int_{0}^{2 \pi} \frac{d \lambda}{2 \pi} \exp \left(i \lambda\left\{a_{2}^{\dagger} a_{2}-a_{1}^{\dagger} a_{1}-(2 K-1)\right\}\right)
$$

is expressed in terms of the canonical coherent states as

$$
\begin{aligned}
\mathbf{I}_{K} & =\int_{0}^{2 \pi} \frac{d \lambda}{2 \pi} e^{-i \lambda(2 K-1)} \sum_{m, n, Q, Q^{\prime}=0}^{\infty} \frac{e^{i \lambda Q}}{m ! n !(m+Q) !\left(n+Q^{\prime}\right) !} \\
& \times \int \frac{\left(d z^{*} d z\right)^{2}}{\pi^{2}} e^{-\mathbf{z}^{\dagger} \mathbf{z}} z_{1}^{m} z_{2}^{m+Q} z_{1}^{* n} z_{2}^{* n+Q^{\prime}} a_{1}^{\dagger} a_{2}^{\dagger m+Q}|(0,0)\rangle\langle(0,0)| a_{1}^{n} a_{2}^{n+Q^{\prime}}
\end{aligned}
$$

A change of variables,

$$
\left(z_{1}, z_{2}\right)=\zeta\left(\frac{\xi}{\sqrt{1-|\xi|^{2}}}, 1\right), \quad\left(d z^{*} d z\right)^{2}=|\zeta|^{2} d \zeta^{*} d \zeta \frac{d \xi^{*} d \xi}{\left(1-|\xi|^{2}\right)^{2}}
$$

and the integration with respect to $\zeta$ leads us to

$$
\begin{aligned}
\mathbf{I}_{K} & =\sum_{m, n=0}^{\infty} \frac{(2 K-1) !(m+n+2 K) !}{m ! n !(m+2 K-1) !(n+2 K-1) !}\left(a_{1}^{\dagger} a_{2}^{\dagger}\right)^{m}|(0,2 K-1)\rangle\langle(0,2 K-1)|\left(a_{1} a_{2}\right)^{n} \\
& \times \int_{D_{(1,1)}} \frac{d \xi^{*} d \xi}{\pi\left(1-|\xi|^{2}\right)^{2}}\left(1-|\xi|^{2}\right)^{2 K+1+(m+n) / 2} \xi^{m} \xi^{* n}
\end{aligned}
$$


Noting that the angular part of the integration in $(\mathrm{B} 25)$ is proportional to $\delta_{m, n}$ and the identity of the beta-function,

$$
\begin{aligned}
& B(p, q) B(p+q, r)=B(q, r) B(q+r, p), \\
& p=2 K+m, q=m+1, r=2 K-1,
\end{aligned}
$$

we obtain

$$
\begin{aligned}
\mathbf{I}_{K}=\sum_{m, n=0}^{\infty} \frac{2 K-1}{\pi} \int_{D_{(1,1)}} & \frac{d \xi^{*} d \xi}{\left(1-|\xi|^{2}\right)^{2}}\left(1-|\xi|^{2}\right)^{2 K} \\
& \times \frac{1}{m !}\left(\xi a_{1}^{\dagger} a_{2}^{\dagger}\right)^{m}|(0,2 K-1)\rangle\langle(0,2 K-1)| \frac{1}{n !}\left(\xi^{*} a_{1} a_{2}\right)^{n} .
\end{aligned}
$$

In view of (B21), $\mathbf{I}_{K}$ is nothing but the resolution of unity in the "spin"- $K$ representation of $S U(1,1)$

$$
\mathbf{I}_{K}=\frac{2 K-1}{\pi} \int \frac{d \xi^{*} d \xi}{\left(1-|\xi|^{2}\right)^{2}}|\xi\rangle\langle\xi|
$$

We cannot expect, however, another type of coherent states in this case; because we are dealing only with one connected component of $S U(1,1)$ and there is no $S U(1,1)$ transformation similar to $(\mathbb{B 1 2})$ leaving the constraint $(\mathbb{B 1 9})$ invariant: for example we may use

$$
\left(z_{1}, z_{2}\right)=\zeta\left(1, \frac{\xi}{\sqrt{1-|\xi|^{2}}}\right), \quad\left(d z^{*} d z\right)^{2}=|\zeta|^{2} d \zeta^{*} d \zeta \frac{d \xi^{*} d \xi}{\left(1-|\xi|^{2}\right)^{2}}
$$

instead of (B24). However it does provide neither $S U(1,1)$ invariant measure nor coherent states in terms of $\xi$ after $\zeta$-integration. Therefore we have only one singularity under the reduction $\mathbf{C}^{2} \rightarrow D_{(1,1)}$ in this case. Following the same idea developed in $S U(2)$ case, we obtain a new expression for the trace formula:

$$
\begin{aligned}
& \operatorname{Tr} e^{-i K_{3} T} \\
= & \lim _{\varepsilon \rightarrow+0} \int_{0}^{2 \pi} \frac{d \lambda}{2 \pi} \int \frac{\left(d z^{*} d z\right)^{2}}{\pi^{2}}\left\langle\mathbf{z}\left|\exp \left\{-i\left(\mathbf{a}^{\dagger} \mathbf{a}+1\right) T / 2+i \lambda\left(-\mathbf{a}^{\dagger} \sigma_{3} \mathbf{a}-(2 K-1)\right)-\varepsilon \mathbf{a}^{\dagger} \mathbf{a}\right\}\right| \mathbf{z}\right\rangle \\
= & e^{-i T / 2} \lim _{\varepsilon \rightarrow+0} \int_{0}^{2 \pi} \frac{d \lambda}{2 \pi} e^{-i(2 K-1) \lambda} \frac{1}{\left(1-e^{i \lambda-\varepsilon-i T / 2}\right)\left(1-e^{-i \lambda-\varepsilon-i T / 2}\right)} \\
= & e^{-i T / 2} \lim _{\varepsilon \rightarrow+0} \oint_{|w|=1} \frac{d w}{2 \pi i} w^{2 K-1} \frac{1}{\left(1-w e^{-\varepsilon-i T / 2}\right)\left(w-e^{-\varepsilon-i T / 2}\right)} \\
= & \frac{e^{-i K T}}{1-e^{-i T}} .
\end{aligned}
$$




\section{REFERENCES}

[1] H. B. Nielsen and D. Rohrlich, Nucl. Phys. B299 (1988) 471.

[2] T. Kashiwa, Int. J. Mod. Phys. A5 (1990) 375.

[3] K. Johnson, Ann. Phys. 192 (1989) 104.

[4] M. Stone, Nucl. Phys. B314, (1989) 557.

E. Keski-Vakkuri, A.J. Niemi, G. Semenoff, and O. Tirkkonen, Phys. Rev. D44 (1991), 3899 .

[5] J. J. Duistermaat and G. J. Heckman, Invent. Math. 69 (1982), 259.

[6] S. G. Rajeev, S. K. Rama, and S. Sen, J. Math. Phys. 35 (1994) 2259.

[7] A. Alekseev, L. D. Faddeev, and S. Shatashvili, J. Geom. Phys. 5 (1989) 391.

[8] T. Suzuki, Nucl. Phys. A 398(1983), 557. T. Fukui, J. Math. Phys. 34 (1993), 4455.

[9] E. Witten, QUANTUM FIELD THEORY AND THE JONES POLYNOMIAL in BRAID GROUP ,KNOT THEORY AND STATISTICAL MECHANICS (World Scientific, Singapore, 1989)

[10] J. R. Klauder and Bo-S. Skagerstam, COHERENT STATES (World Scientific, Singapore, 1985).

[11] B. G. Wybourne, CLASSICAL GROUPS FOR PHYSISTS (John Wiley \& Sons, Inc. 1974), page 146.

[12] K.Funahashi, T.Kashiwa, S.Sakoda, and K.Fujii in preparation.

[13] E. Witten, Nucl. Phys. B311 (1988) 46.

[14] J. Schwinger, ON ANGULAR MOMENTUM in QUANTUM THEORY OF ANGULAR MOMENTUM(Academic press, New York, 1965) 


\section{FIGURES}

FIG. 1. Contours of $z$-integration for $S U(2)$ character formula. The original contour $C$ starts

from the origin and ends at a point in finite distance. This is the consequence of compactness of $S U(2)$.

FIG. 2. Contours of $z$-integration for $S U(1,1)$ trace formula. The original contour $C$ starts from the origin and never returns to a point in finite distance. This is the consequence of non-compactness of $S U(1,1)$. 
Fig. 1.

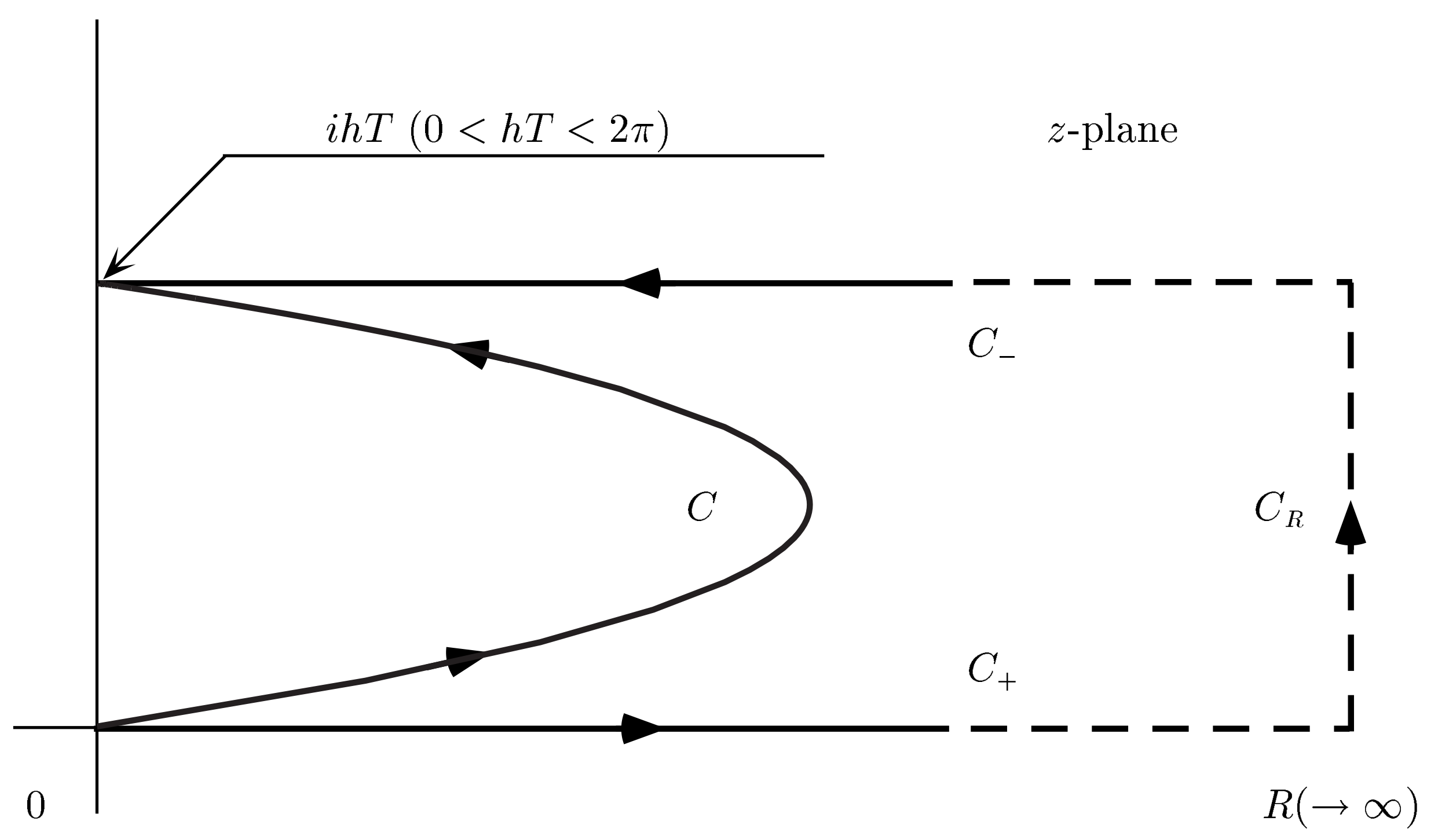


Fig. 2.

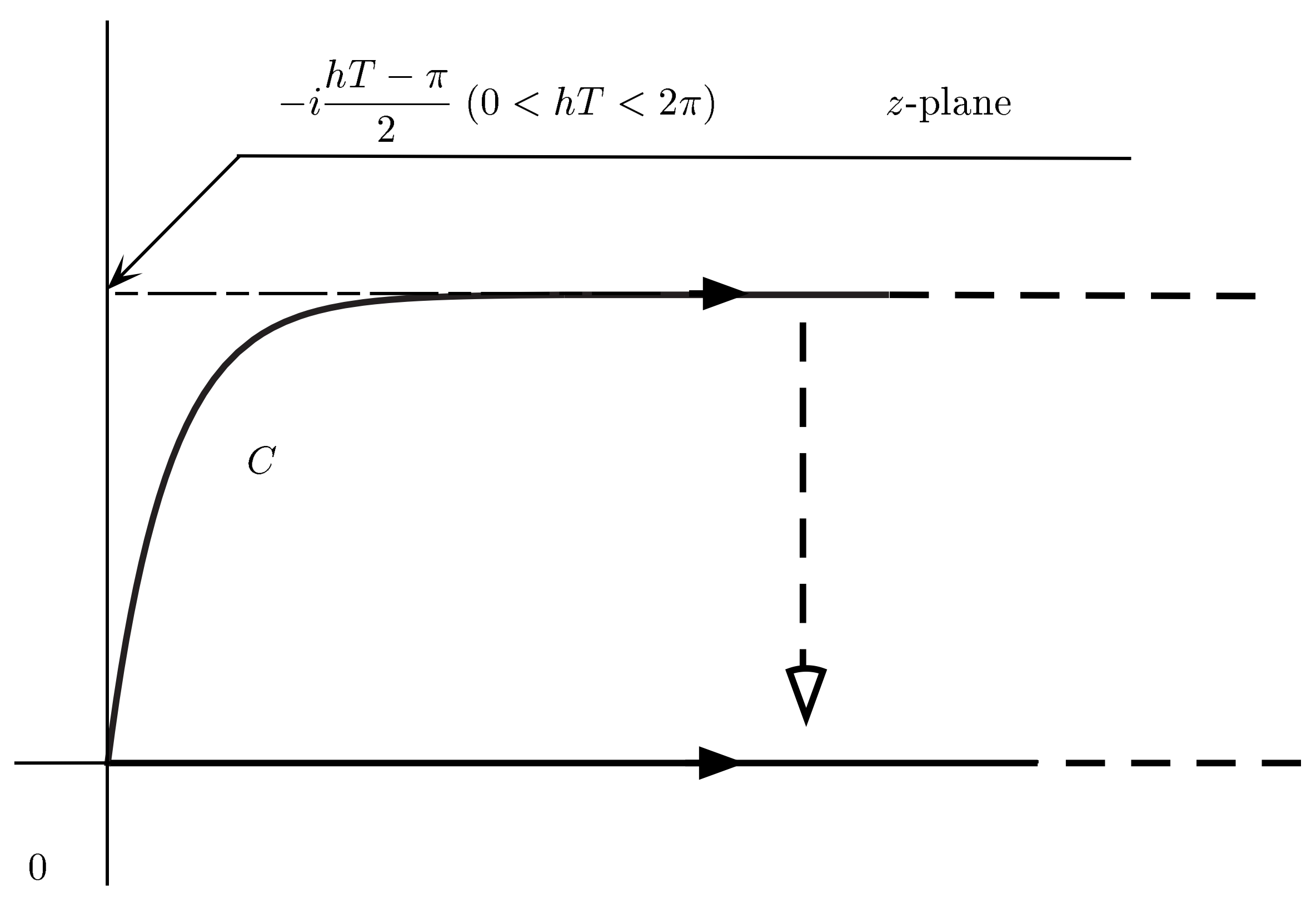


This figure "fig1-1.png" is available in "png" format from: http://arxiv.org/ps/hep-th/9409116v2 
This figure "fig2-1.png" is available in "png" format from: http://arxiv.org/ps/hep-th/9409116v2 